\title{
Primary tumor or metastasis? Extremely rare fibrosarcoma of the left atrial appendage
}

Marco Zanobini, MD, PhD, ${ }^{\mathrm{a}}$ Silvia Travaglini, MD, ${ }^{\mathrm{a}}$ Mauro Pepi, MD, PhD, ${ }^{\mathrm{b}}$ and Matteo Saccocci, MD, ${ }^{\mathrm{a}, \mathrm{c}}$ Milan, Italy, and Zürich, Switzerland

\footnotetext{
From the Departments of ${ }^{\mathrm{a} C a r d i o v a s c u l a r}$ Surgery and ${ }^{\mathrm{b}}$ Cardiac Imaging, IRCCS Centro Cardiologico Monzino, Milan, Italy; and ${ }^{\mathrm{c}}$ Department of Cardiovascular Surgery, Heart Center Zürich, University Hospital of Zürich, University of Zürich, Zürich, Switzerland.

Disclosures: Authors have nothing to disclose with regard to commercial support.

Received for publication Oct 6, 2017; revisions received April 15, 2018; accepted for publication April 18, 2018; available ahead of print May 18, 2018.

Address for reprints: Matteo Saccocci, MD, Department of Cardiac Surgery, Centro Cardiologico Monzino, IRCCS, Via C. Parea 4, 20138 Milan, Italy (E-mail: matteo.saccocci@unimi.it).

J Thorac Cardiovasc Surg 2018;156:e153-6

$0022-5223 / \$ 36.00$

Copyright (C) 2018 by The American Association for Thoracic Surgery

https://doi.org/10.1016/j.jtcvs.2018.04.084
}

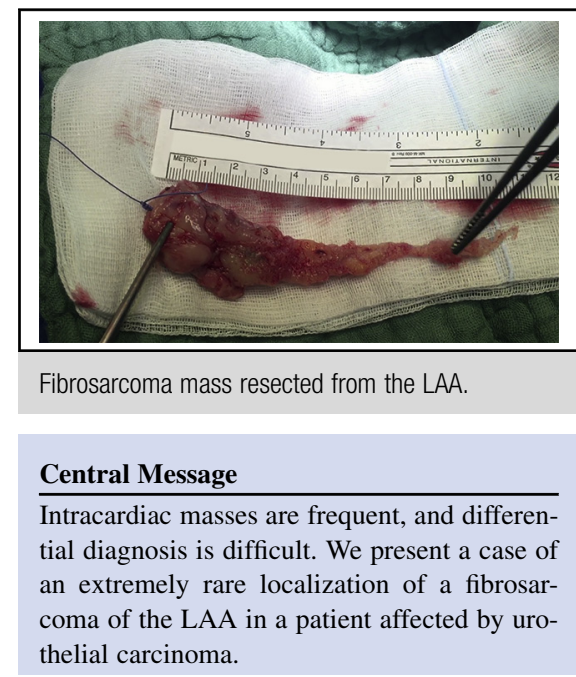

See Editorial Commentary page e 157.
Video clip is available online.

A 49-year-old man was transferred to the Department of Cardiac Surgery from the Oncology Division after echocardiographic evidence of an intracardiac mass. Four months previously, he underwent transurethral resection for a T3grade invasive urothelial bladder carcinoma (transitional cell carcinoma). At that time, a full-body computed tomography (CT) scan was performed with evidence of multiple metastases at the level of lungs and bones, but no cardiac infiltrations were reported, making him a good candidate for a first-line chemotherapy protocol. After 3 months, during planned pretreatment screening, a transthoracic echocardiography revealed a huge left atrial intracardiac mass.

The patient was promptly transferred to our department for further investigations and treatment. At admittance, he had no cardiopulmonary symptoms (New York Heart Association class I), electrocardiogram and blood pressure were normal, and there were no laboratory test alterations. Transesophageal echocardiography showed a hyperechoic huge mass with a maximum diameter of $7 \mathrm{~cm}$ originating from the left atrial appendage (LAA) characterized by high mobility and complete engagement of the mitral valve up to the apical portion of the left ventricle (LV) (Figure 1 and Video 1). An urgent electrocardiogram-gated cardiac CT scan was performed showing a dilated LAA (maximum diameter: $5.3 \mathrm{~mm}$ ) with a huge mass entirely filling the LAA cavity (Figure 1). The neoformation originated in the LAA, crossed the mitral valve, and floated into the
LV. Its distal portion was highly mobile with irregular appendages swirling into the LV cavity. There was no evidence of pulmonary vein involvement. CT scan and echocardiographic images offered an ambiguous description of the intracardiac mass, leading to a difficult differential diagnosis between thrombotic formation and tumor. The patient's clinical history was highly suggestive for urothelial metastasis, but to confirm this hypothesis and to exclude other pathologies (intracardiac thrombotic formation or different primary tumor ${ }^{1}$ ), we proceeded with contrastenhanced cardiovascular magnetic resonance imaging to improve characterization of the mass (analysis of structure and vascularization) (Figure 1 and Video 2). The cine imaging by steady-state free-precession sequence showed an extremely mobile neoformation of $43 \times 20 \mathrm{~mm}$, originating from the left appendage, which was entirely filled by the mass. It engaged the mitral valve in proximity of the left anterior leaflet reaching the mid portion of the LV. It appeared iso-intense in double-inversion recovery T1weighted sequences (Figure 1, B) and nonhomogeneously hyper-intense in the black-blood T2-weighted short inversion-time inversion-recovery sequences (Figure 1, $C)$. On first-pass perfusion, the mass did not show any contrast, but late gadolinium hyperenhancement showed increased signal after 3, 5, and 7 minutes, respectively. Therefore, magnetic resonance imaging findings clarified the neoplastic nature of the mass and excluded an 


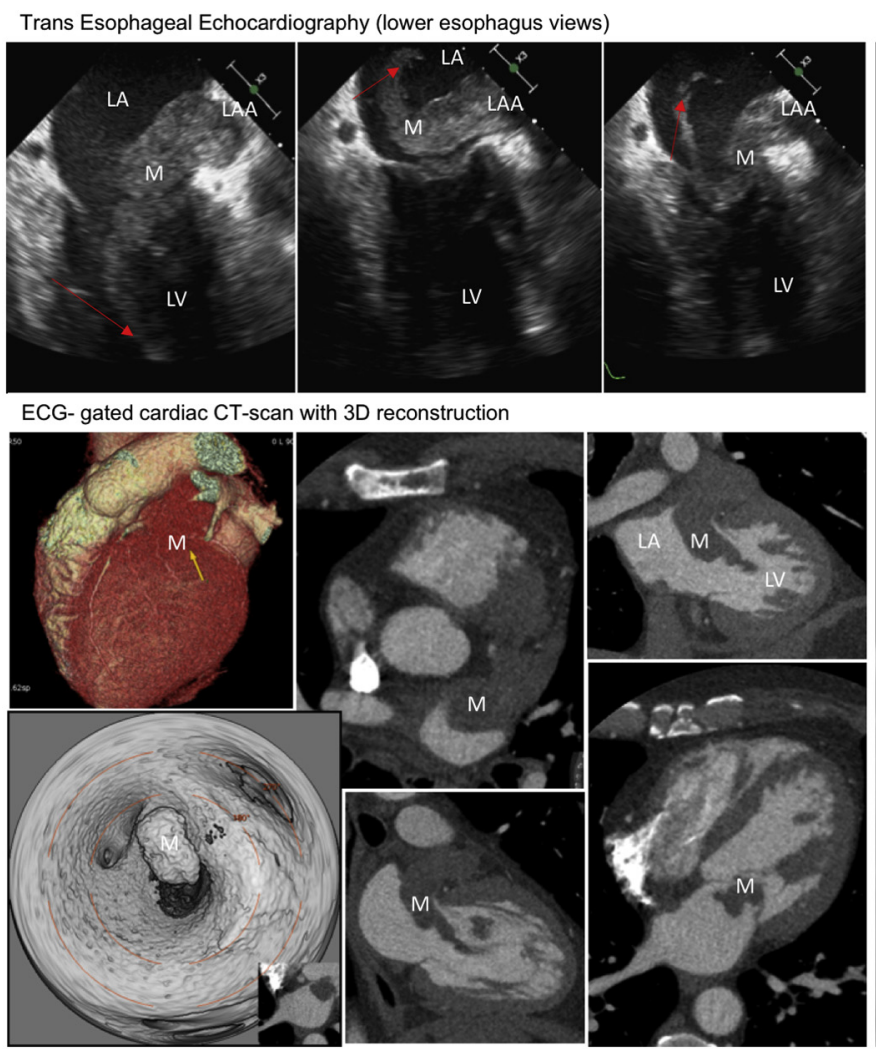

Contrast-enhanced cardiovascular magnetic resonance (MRI)

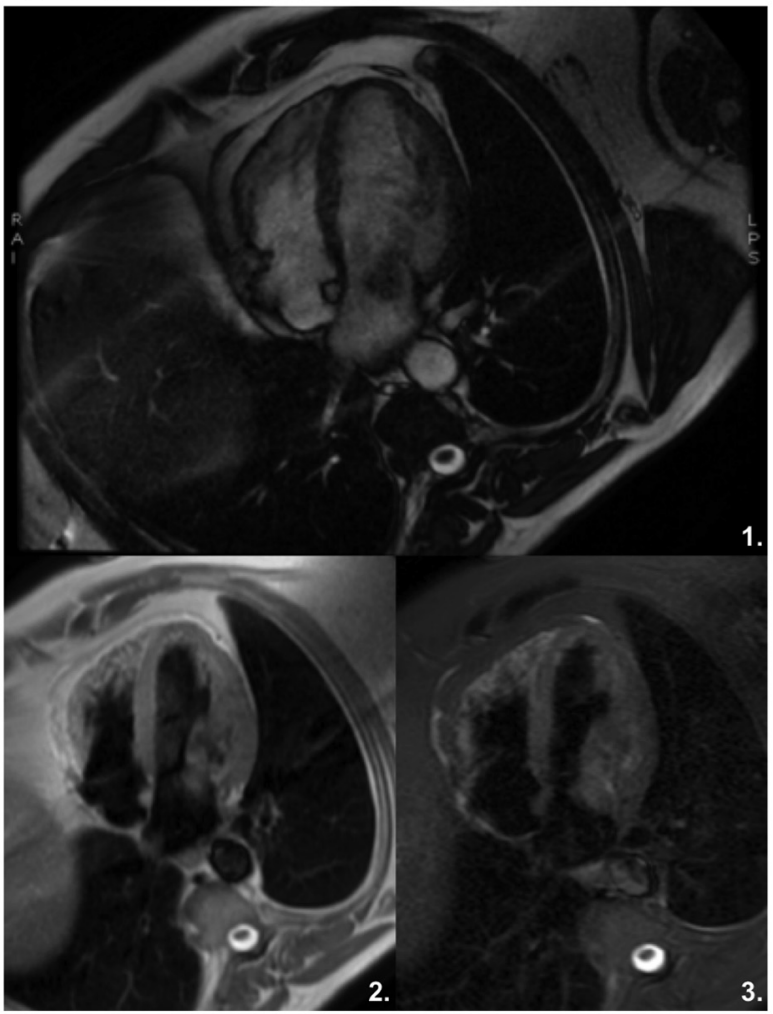

FIGURE 1. Transesophageal echocardiography of the lower esophagus shows a huge intracardiac mass originating from the LAA, cyclically crossing the mitral valve, and swirling into the left atrium and LV cavities; electrocardiogram-gated cardiac CT scan with 3-dimensional reconstruction. Contrastenhanced cardiovascular magnetic resonance (Discovery 450, GE Healthcare [Chicago, Ill], 1,5 T). Panel 1: steady-state (steady-state free precession cine imaging) extremely mobile neoformation of $43 \times 20 \mathrm{~mm}$, originating from the LAA, which was entirely filled by the mass. It engaged the mitral valve in proximity of the left anterior leaflet reaching the mid-portion of the LV. Panel 2: T1-weighted sequences. Panel 3: T2-Tir sequences. Red arrow highlights the distal mobile part of the neoformation. $L A$, Left atrium; $L A A$, left atrial appendage; $M$, mass; $L V$, left ventricle; $E C G$, electrocardiogram; $C T$, computed tomography; 3D, 3-dimensional.

intracardiac thrombotic formation. On this basis, the heart team decided on urgent resection. With the patient under general anesthesia, we proceeded to central cardiopulmonary bypass implantation through a complete median sternotomy. After pericardium incision, we noticed a moderate pericardial effusion, and a sample was sent to laboratory for histopathologic and cytologic tests. Having performed a left atriotomy through the Waterston's, we immediately faced the atrial part of the mass. We had a complete visualization of the tumor without opening the transverse sinus. It occupied the entire LAA, and passing through the mitral valve, it reached the distal portion of the LV. We made a complete excision of the mass (Figure 2) and the LAA. A complete freezing of resection margins (Frigitronics Cardiac Cryosurgical System; Atricure Inc, Mason, Ohio) of the appendage surgical neck was performed to ensure a better sterilization from possible residual cancer cells. To close the iatrogenic large atrial wall defect, we used a pericardial patch strengthened by 2 separate Teflon bands. The mass was sent to the laboratory for histologic diagnosis. The patient experienced no perioperative complication during the in-hospital stay, and he was transferred on postoperative day 6 to the rehabilitation unit.

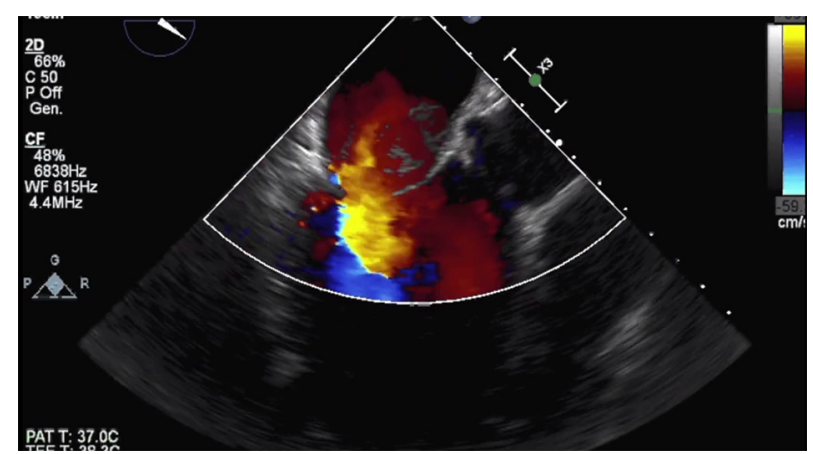

VIDEO 1. Preoperative transthoracic echocardiography showing a huge intracardiac mass originating from the LAA, cyclically crossing the mitral valve, and swirling into the left atrium and LV cavities. Video available at: https://www.jtcvs.org/article/S0022-5223(18)31222-4/fulltext. 


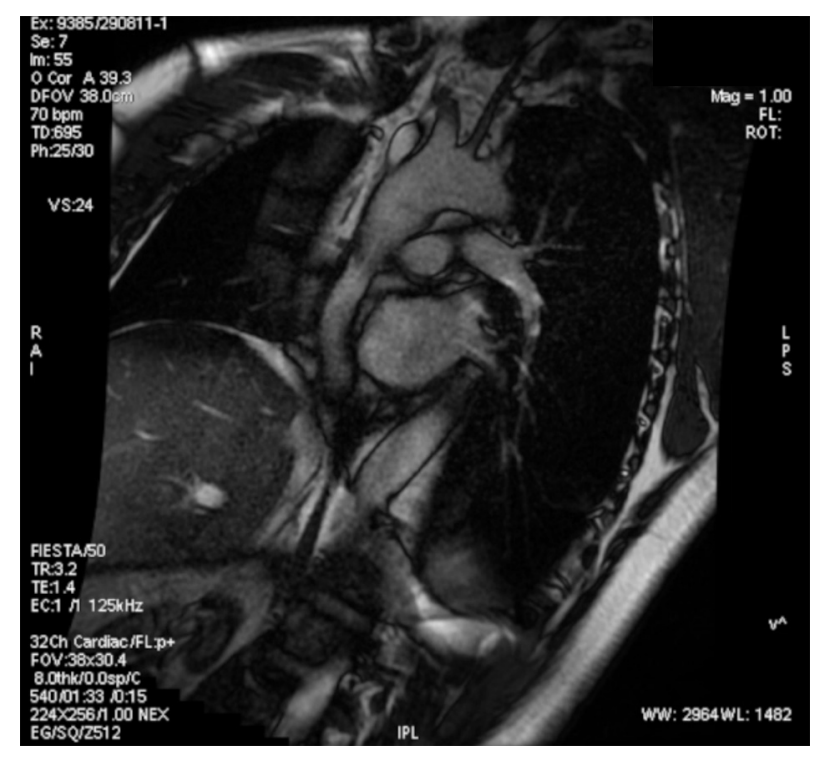

VIDEO 2. Magnetic resonance imaging of the neoformation. Video available at: https://www.jtcvs.org/article/S0022-5223(18)31222-4/fulltext.

Predischarge transthoracic echocardiography showed the absence of residual intracardiac mass and normal mitral and LV function. The laboratory report of the pericardial fluid sample showed the presence of abnormal cells suggestive of transitional cell carcinoma dissemination. The real surprise came from the histologic analysis of the mass: primary cardiac tumor, a grade 3 fibrosarcoma $^{2}$ (Figure 2), and resection margins negative for malignancy. At 3 months follow-up, the patient was asymptomatic without evidence of cardiac recurrence. $\mathrm{He}$ is still in treatment and follow-up according to the therapeutic chemotherapy protocol for bone, lung, and pericardial metastasis of transitional cell carcinoma and for LAA fibrosarcoma.

The authors want to thank the Department of Cardiac Surgery and Cardiac Imaging, IRCCS Centro Cardiologico Monzino, Milan, Italy, and the Department of Anato-pathology, IEO Milan, Milan, Italy. In particular, the authors thank Saima Mushtaq, MD, $\mathrm{PhD}$; Laura Cavallotti, MD; Francesca Susini, MD; Fausto Maffini, MD; Elisa De Camilli, MD; and Andrea Daniele Annoni, MD.

\section{References}

1. Lee WC, Huang MP, Fu M. Multiple intracardiac masses: myxoma, thrombus or metastasis: a case report. J Med Case Rep. 2015;9:179.

2. Burke A, Tavora F. The 2015 WHO classification of tumors of the heart and pericardium. J Thorac Oncol. 2016;11:441-52. 




FIGURE 2. Panel 1, Intraoperative findings. Panel 2, Histologic diagnosis of grade 3 fibrosarcoma: hematoxylin-eosin staining at $10 \times$ and $40 \times($ A and B, respectively). Vimentin staining at $10 \times$ and $40 \times(\mathrm{C}$ and $\mathrm{D}$, respectively). 